\title{
APPLICATION OF THE METHOD OF BOUNDARY INTEGRAL EQUATIONS FOR NON-STATIONARY PROBLEM OF THERMAL CONDUCTIVITY IN AXIALLY SYMMETRIC DOMAIN
}

\author{
Grigoriy Zrazhevsky \\ Department of Theoretical Mechanics \\ Taras Shevchenko National University of Kyiv \\ 64 Volodymyrs'ka str., Kyiv, Ukraine, 01033 \\ zrazhevsky@aorda.com \\ Vera Zrazhevska \\ Department of Differential Equations \\ National Technical University of Ukraine "Igor Sikorsky Kyiv Polytechnic Institute" \\ 37 Peremogy ave., Kyiv, Ukraine, 03056 \\ vera.zrazhevska@gmail.com
}

\begin{abstract}
The article considers the non-stationary initial-boundary problem of thermal conductivity in axially symmetric domain in Minkowski space, formulated as equivalent boundary integral equation. Using the representation of the solution in the form of a Fourier series expansion, the problem is reformulated as an infinite system of two-dimensional singular integral equations regarding expansion coefficients. The paper presents and investigates the explicit form for fundamental solutions used in the integral representation of the solution in the domain and on the border. The obtained results can be used in the construction of efficient numerical boundary element method for estimation of structures behavior under the influence of intense thermal loads in real-time.

Keywords: initial-boundary problem of thermal conductivity, boundary integral equation, axially symmetric domain, fundamental solution for axially symmetric domain.

\section{Introduction}

Large number of studies is devoted to the theory of temperature stresses and engineering methods for their calculation [1-4]. Mathematical model of thermal stresses of metal structures is reduced to calculation of non-stationary behavior of thermal fields by solving a non-stationary initial-boundary value problem of thermal conductivity, and calculation of temperature stresses by solving stationary boundary problem of elasticity $[1,2]$. Basic analytical solutions for model problems for canonical domains are obtained [1-3]. At the same time, calculations and control of real structures require the development of efficient numerical methods that allow to calculate the behavior of structures under the influence of intense thermal loads in real-time. One of these methods is the boundary element method that is one of numerous implementations of the method of boundary integral equations [5-7].

The method of boundary integral equations is based on the transition from the non-stationary initial-boundary value problem of thermal conductivity to the equivalent boundary integral equation. This approach has numerous advantages over classical methods because it allows to reduce the dimension of the problem, the obtained solution is semi-analytical and therefore it can be analyzed with analytical methods. The method uses the Green function as a fundamental solution that allows to satisfy one part of the boundary conditions and the condition at infinity (in the case of infinity inclusion) automatically. The method allows to build special boundary elements, that take into account the asymptotic behavior of the solution in a neighborhood of singular points of the problem $[6,7]$.

Special numerical methods, that take into account some particular qualities of constructions, are more effective in comparison with universal methods. Since cylindrical structures, such as pipelines, reactor vessels, constitute a significant share of production elements, calculation of 
critical temperature modes of their operation is very important. Axially symmetric domain allows to use the representation of the solution in the form of a Fourier series expansion.

In practical applications it is important to take into account a finite number of harmonics only, so this problem can be reduced to a finite system of two-dimensional (spatial domain and time) boundary singular integral equations.

\section{Materials and methods}

\section{1. Previous results}

Let's introduce the following notations: $\mathrm{x}=\left(\mathrm{x}_{1}, \mathrm{x}_{2}, \mathrm{x}_{3}\right) \in \mathrm{R}^{3}, \Omega \subset \mathrm{R}^{3}$ is a space domain with the boundary surface $\Gamma=\Gamma_{\mathrm{u}} \cup \Gamma_{\mathrm{q}}$, $\mathrm{t}$ is time; $\mathrm{u}(\mathrm{x}, \mathrm{t})$ is a temperature at a point $\mathrm{x}$ at a time moment $\mathrm{t} ; \mathrm{c}(\mathrm{x})$ is the heat capacity, $\mathrm{k}(\mathrm{x})$ is the thermal conductivity; $\rho(\mathrm{x})$ is the matter density, $f(x, t)$ is the intensity of heat sources at a point $x$ at a time moment $t$.

Let's consider the problem for the heat equation:

$$
\operatorname{c\rho } \frac{\partial \mathrm{u}}{\partial \mathrm{t}}(\mathrm{x}, \mathrm{t})=\operatorname{div}(\mathrm{kgrad} u(x, t))+f(x, t), x \in \Omega, t>t_{0}
$$

with the boundary conditions:

$$
\left\{\begin{array}{l}
\mathrm{u}(\mathrm{x}, \mathrm{t})=\overline{\mathrm{u}}(\mathrm{x}, \mathrm{t}), \mathrm{x} \in \Gamma_{\mathrm{u}}, \mathrm{t} \geq \mathrm{t}_{0}, \\
\mathrm{q}(\mathrm{x}, \mathrm{t})=\overline{\mathrm{q}}(\mathrm{x}, \mathrm{t}), \mathrm{x} \in \Gamma_{\mathrm{q}}, \mathrm{t} \geq \mathrm{t}_{0}
\end{array}\right.
$$

and initial condition:

$$
\mathrm{u}\left(\mathrm{x}, \mathrm{t}_{0}\right)=\mathrm{u}_{0}(\mathrm{x}), \mathrm{x} \in \bar{\Omega},
$$

where $\bar{\Omega}=\Omega \cup \Gamma, \mathrm{q}(\mathrm{x}, \mathrm{t})=\mathrm{k} \frac{\partial \mathrm{u}}{\partial \overrightarrow{\mathrm{n}}}, \overrightarrow{\mathrm{n}}$ is an external normal to the surface.

Let's consider the initial boundary problem (1)-(3) as a boundary problem in Minkowski space $(x, t)$, where $x$ is a point of three-dimensional space, $t$ is time. Obviously, we get the boundary problem for cylinder $\left(\mathrm{x} \in \Omega, \mathrm{t}>\mathrm{t}_{0}\right)$ with boundary conditions: (2) on the boundary surface of the cylinder $\left(\mathrm{x} \in \Gamma_{\mathrm{u}} \cup \Gamma_{\mathrm{q}}, \mathrm{t} \geq \mathrm{t}_{0}\right)$ and (3) on the cylinder base.

In [8], using the method of weighted residuals $[9,10]$, the integral representation of the solution is obtained and its correctness is proved, the conditions on the parameters of the passage to the limit for correct interpretation of the singular integral in four-dimensional space are formulated.

The article [8] presents the integral equivalent of the problem:

$$
\begin{gathered}
\chi_{\left(\Omega, \mathrm{t}>\mathrm{t}_{0}\right)}(\xi, \tau) \cdot \mathrm{u}(\xi, \tau)+\int_{\mathrm{t}_{0}}^{\tau} \mathrm{dt} \int_{\Gamma}(\mathrm{u} *(\xi-\mathrm{x}, \tau-\mathrm{t}) \mathrm{q}(\mathrm{x}, \mathrm{t})-\mathrm{u}(\mathrm{x}, \mathrm{t}) \mathrm{q} *(\mathrm{x}-\xi, \tau-\mathrm{t})) \mathrm{d} \Gamma_{\mathrm{x}}= \\
=\int_{\Omega} \rho \mathrm{cu} \mathrm{u}_{0}(\mathrm{x}) \mathrm{u} *\left(\xi-\mathrm{x}, \tau-\mathrm{t}_{0}\right) \mathrm{d} \Omega_{\mathrm{x}}-\int_{\mathrm{t}_{0}}^{\tau} \mathrm{dt} \int_{\Omega} \mathrm{f}(\mathrm{x}, \mathrm{t}) \mathrm{u} *(\xi-\mathrm{x}, \tau-\mathrm{t}) \mathrm{d} \Omega_{\mathrm{x}},
\end{gathered}
$$

where $\mathrm{H}$ is the Heaviside function, $\mathrm{u}^{*}$ is a fundamental solution of the heat equation that satisfies the principle of causality and is limited at infinity for four-dimensional Minkowski space [8]:

$$
\begin{gathered}
\mathrm{u}^{*}=-\frac{(\rho \mathrm{c})^{1 / 2}}{(4 \pi \mathrm{k}(\tau-\mathrm{t}))^{3 / 2}} \exp \left(-\frac{|\xi-\mathrm{x}|^{2} \rho \mathrm{c}}{4 \mathrm{k}(\tau-\mathrm{t})}\right) \mathrm{H}(\tau-\mathrm{t}), \\
\chi_{\left(\Omega, \mathrm{t}>\mathrm{t}_{0}\right)}(\xi, \mathrm{t})=\left\{\begin{array}{l}
1,(\xi, \mathrm{t}) \in\left(\Omega, \mathrm{t}>\mathrm{t}_{0}\right) \\
0,(\xi, \mathrm{t}) \notin\left(\Omega, \mathrm{t}>\mathrm{t}_{0}\right)
\end{array}\right.
\end{gathered}
$$

is the area function (indicator). 
Obviously, (5) is the quasi equation. It allows to determine $\mathrm{u}(\mathrm{x}, \mathrm{t})$ at any point inside the area $\left(x \in \Omega, t>t_{0}\right)$ if values of functions $u(x, t)$ and $q(x, t)$ on $\Gamma$ are known. So, the problem is to determine $\mathrm{u}(\mathrm{x}, \mathrm{t})$ on $\Gamma_{\mathrm{q}}$ and $\mathrm{q}(\mathrm{x}, \mathrm{t})$ on $\Gamma_{\mathrm{u}}$. To get a boundary integral equation in [8] the limit transition in (8) as the point of source approaches the boundary of the four-dimensional domain $\xi \rightarrow \bar{\xi} \in \Gamma$ was conducted, the interpretation of the Cauchy principal value of integral singularity $\int_{\mathrm{t}_{0}}^{\tau} \mathrm{dt} \int_{\Gamma} \mathrm{u}(\mathrm{x}, \mathrm{t}) \mathrm{q}^{*}(\mathrm{x}-\bar{\xi}, \tau-\mathrm{t}) \mathrm{d} \Gamma_{\mathrm{x}}$ was fulfilled, the conditions on the parameters of the passage to the ${ }_{\text {limit }}^{\mathrm{t}_{0}} \Gamma$ for its correct interpretation were formulated.

The boundary integral equation of the problem was obtained in the form:

$$
\begin{gathered}
\mathrm{C}(\bar{\xi}) \mathrm{u}(\bar{\varepsilon}, \tau)+\int_{\mathrm{t}_{0}}^{\tau} \mathrm{dt} \int_{\Gamma}(\mathrm{u} *(\bar{\xi}-\mathrm{x}, \tau-\mathrm{t}) \mathrm{q}(\mathrm{x}, \mathrm{t})-\mathrm{u}(\mathrm{x}, \mathrm{t}) \mathrm{q} *(\bar{\xi}-\mathrm{x}, \tau-\mathrm{t})) \mathrm{d} \Gamma_{\mathrm{x}}= \\
=\int_{\Omega} \rho \mathrm{c} \mathrm{u}_{0}(\mathrm{x}) \mathrm{u} *\left(\bar{\xi}-\mathrm{x}, \tau-\mathrm{t}_{0}\right) \mathrm{d} \Omega_{\mathrm{x}}-\int_{\mathrm{t}_{0}}^{\tau} \mathrm{dt} \int_{\Omega} \mathrm{f}(\mathrm{x}, \mathrm{t}) \mathrm{u} *(\bar{\xi}-\mathrm{x}, \tau-\mathrm{t}) \mathrm{d} \Omega_{\mathrm{x}}, \bar{\xi} \in \Gamma, \mathrm{t}_{0}<\mathrm{t}<\tau,
\end{gathered}
$$

where $C(\bar{\xi})=\frac{1}{4 \pi}\left(4 \pi-S\right.$ ) (if $\bar{\xi}$ is regular then $C(\bar{\xi})=\frac{1}{2}$ ) and the second integral in the left side is taken in the sense of principal value.

Thus, the original problem (1) - (3) was reduced to solving of the singular integral equation (5), that is, to finding $\mathrm{u}(\mathrm{x}, \mathrm{t})$ for $\mathrm{x} \in \Gamma_{\mathrm{q}}$ and $\mathrm{q}(\mathrm{x}, \mathrm{t})$ for $\mathrm{x} \in \Gamma_{\mathrm{u}}$. Then the integral representation of the solution (4) can be used to find $u(\xi, \tau)$ for $(\xi, \tau) \in\left(\Gamma, \tau>t_{0}\right)$. It should be emphasized that (5) is an equation in three-dimensional space $\left(\bar{\xi} \in \Gamma, \tau>\mathrm{t}_{0}\right)$, and not in four-dimensional space $\left(\xi \in \mathrm{R}, \tau>\mathrm{t}_{0}\right)$ as the original problem. So, the transition to the boundary integral equation allowed to reduce the dimension of the problem [8].

\section{2. A linear boundary integral equation for the axially symmetric domain}

Let the spatial domain $\Omega$ with the border $\Gamma$ is axisymmetrical, but the boundary and initial conditions are not axisymmetrical. We show that in this case the boundary integral equation (5) can be reduced to an infinite countable set of singular integral equations that are two-dimensional in Minkowski space.

Let's introduce the spatial cylindrical coordinate systems $\left(r_{x}, \theta_{x}, z_{x}\right)$ in the space $x$ and $\left(\mathrm{r}_{\xi}, \theta_{\xi}, \mathrm{z}_{\xi}\right)$ in the space $\xi$. ( $\mathrm{x}$ is an observation point, $\xi$ is a point of source). As $\Gamma$ is the surface of rotating, all limit functions in (5) can be expanded in Fourier series:

$$
\begin{aligned}
& f(x)=\sum_{n=0}^{\infty}\left[f_{n}^{c}(\bar{x}) \cos n \theta_{x}+f_{n}^{s}(\bar{x}) \sin n \theta_{x}\right], \bar{x}=\left(r_{x}, z_{x}\right) \in \bar{\Gamma} \\
& \left\{\begin{array}{l}
\mathrm{f}_{\mathrm{n}}^{\mathrm{c}}(\overline{\mathrm{x}})=\frac{1}{\pi} \int_{0}^{2 \pi} \mathrm{f}\left(\mathrm{r}_{\mathrm{x}}, \theta_{\mathrm{x}}, \mathrm{z}_{\mathrm{x}}\right) \cos n \theta_{\mathrm{x}} \mathrm{d} \theta_{\mathrm{x}}, \mathrm{n}=1,2, \ldots \\
\mathrm{f}_{0}^{\mathrm{c}}(\overline{\mathrm{x}})=\frac{1}{\pi} \int_{0}^{2 \pi} \mathrm{f}\left(\mathrm{r}_{\mathrm{x}}, \theta_{\mathrm{x}}, \mathrm{z}_{\mathrm{x}}\right) \mathrm{d} \theta_{\mathrm{x}} \\
\mathrm{f}_{\mathrm{n}}^{\mathrm{s}}(\overline{\mathrm{x}})=\frac{1}{\pi} \int_{0}^{2 \pi} \mathrm{f}\left(\mathrm{r}_{\mathrm{x}}, \theta_{\mathrm{x}}, \mathrm{z}_{\mathrm{x}}\right) \sin \mathrm{n} \theta_{\mathrm{x}} \mathrm{d} \theta_{\mathrm{x}}, \mathrm{n}=1,2, \ldots
\end{array}\right.
\end{aligned}
$$

Let's substitute decompositions like (6) for functions $\mathrm{u}(\mathrm{x}, \mathrm{t}), \mathrm{q}(\mathrm{x}, \mathrm{t})$ to (5), multiply by $\cos \mathrm{k} \theta_{\xi}, \sin \mathrm{k} \theta_{\xi}$ and integrate over $\theta_{\xi}$ in the interval $[0,2 \pi]$. Because of the orthogonality of trigonometric functions it is easy to see that:

$$
\left\{\begin{array}{l}
\frac{1}{\pi} \int_{0}^{2 \pi} \sum_{\mathrm{k}=0}^{\infty}\left\{\mathrm{u}_{\mathrm{k}}^{\mathrm{c}}(\bar{\xi}, \tau) \cos \mathrm{k} \theta_{\mathrm{x}}+\mathrm{u}_{\mathrm{k}}^{\mathrm{s}}(\bar{\xi}, \tau) \sin \mathrm{k} \theta_{\mathrm{x}}\right\} \cos \mathrm{n} \theta_{\xi} \mathrm{d} \theta_{\xi}=\mathrm{u}_{\mathrm{n}}^{\mathrm{c}}(\bar{\xi}, \tau), \\
\frac{1}{\pi} \int_{0}^{2 \pi} \sum_{\mathrm{k}=0}^{\infty}\left\{\mathrm{u}_{\mathrm{k}}^{\mathrm{c}}(\bar{\xi}, \tau) \cos \mathrm{k} \theta_{\mathrm{x}}+\mathrm{u}_{\mathrm{k}}^{\mathrm{s}}(\bar{\xi}, \tau) \sin \mathrm{k} \theta_{\mathrm{x}}\right\} \sin \mathrm{n} \theta_{\xi} \mathrm{d} \theta_{\xi}=\mathrm{u}_{\mathrm{n}}^{\mathrm{s}}(\bar{\xi}, \tau) .
\end{array}\right.
$$


Let's consider the typical expressions in (5). Using the expression of the element of integration $\mathrm{d} \Gamma_{\mathrm{x}}$ in the cylindrical coordinate system $\mathrm{d} \Gamma_{\mathrm{x}}=\mathrm{r}_{\mathrm{x}} \mathrm{d} \theta_{\mathrm{x}} \mathrm{d} \bar{\Gamma}\left(\mathrm{r}_{\mathrm{x}}, \mathrm{z}_{\mathrm{x}}\right)$ we move from the integrals over the domain to integrals over the boundary $\bar{\Gamma}$. Then we have:

$$
\begin{gathered}
\int_{\Gamma} \mathrm{u}^{*}(\xi, \mathrm{x}, \tau, \mathrm{t}) \mathrm{q}(\mathrm{x}, \mathrm{t}) \mathrm{d} \Gamma_{\mathrm{x}}=\int_{0}^{2 \pi} \mathrm{d} \theta_{\mathrm{x}} \int_{\bar{\Gamma}} \mathrm{u}^{*}(\xi, \mathrm{x}, \tau, \mathrm{t}) \mathrm{q}(\overline{\mathrm{x}}, \mathrm{t}) \mathrm{r}_{\mathrm{x}} \mathrm{d} \bar{\Gamma}\left(\mathrm{r}_{\mathrm{x}}, \mathrm{z}_{\mathrm{x}}\right)= \\
=\int_{0}^{2 \pi} \mathrm{d} \theta_{\mathrm{x}} \int_{\bar{\Gamma}} \mathrm{u}^{*}\left(\bar{\xi}, \theta_{\xi}, \bar{x}, \theta, \tau, \mathrm{t}\right) \mathrm{q}(\overline{\mathrm{x}}, \mathrm{t}) \mathrm{r}_{\mathrm{x}} \mathrm{d} \bar{\Gamma}\left(\mathrm{r}_{\mathrm{x}}, \mathrm{z}_{\mathrm{x}}\right)
\end{gathered}
$$

and so

$$
\begin{gathered}
\frac{1}{\pi} \int_{0}^{2 \pi} \cos n \theta_{\xi} d \theta_{\xi} \int_{\Gamma} u^{*}(\xi, x, \tau, t) q(x, t) d \Gamma_{x}= \\
=\int_{0}^{2 \pi} d \theta_{x} \int_{\Gamma} \frac{1}{\pi} \int_{0}^{2 \pi} \cos n \theta_{\xi} u^{*}\left(\bar{\xi}, \theta_{\xi}, \bar{x}, \theta_{x}, \tau, t\right) d \theta_{\xi} q\left(\bar{x}, \theta_{x}, t\right) r_{x} d \bar{\Gamma}\left(r_{x}, z_{x}\right) .
\end{gathered}
$$

Taking into account, that

$$
\begin{gathered}
u^{*}(\xi-x, \tau-t)=u^{*}(|\xi-x|, \tau-t), \\
|x-\xi|^{2}=r_{x}^{2}+r_{\xi}^{2}-2 r_{x} r_{\xi} \cos \left(\theta_{\xi}-\theta_{x}\right)+\left(z_{\xi}-z_{x}\right)^{2},
\end{gathered}
$$

we have: $\mathrm{u}^{*}(|\xi-\mathrm{x}|, \tau-\mathrm{t})=\mathrm{u}^{*}(\bar{\xi}, \overline{\mathrm{x}}, \theta, \tau-\mathrm{t})$, where $\theta=\theta_{\mathrm{x}}-\theta_{\xi}$. Thus, we have shown that $\mathrm{u}^{*}$ is independent separately from $\theta_{\mathrm{x}}, \theta_{\xi}$ and depends only on their difference $\theta_{\mathrm{x}}-\theta_{\xi}$. This allows us to move from the integral over $\theta_{x}$ or $\theta_{\xi}$ to the integral over $\theta=\theta_{x}-\theta_{\xi}$.

Taking into account that $\cos n \theta_{\xi}=\cos n\left(\theta_{x}-\theta\right)$ and presented $\bar{q}\left(\bar{x}, \theta_{x}, t\right)$ in the form (6), we rewrite (9) as:

$$
\begin{gathered}
\frac{1}{\pi} \int_{0}^{2 \pi} \cos n \theta_{\xi} d \theta_{\xi} \int_{\Gamma} u^{*}(\xi, x, \tau, t) q(x, t) d \Gamma_{x}= \\
=\int_{\bar{\Gamma}}\left[u_{n}^{*_{c}}(\bar{\xi}, \bar{x}, \tau-t) q_{n}^{c}(\bar{x}, t)+u_{n}^{*_{s}}(\bar{\xi}, \bar{x}, \tau-t) q_{n}^{s}(\bar{x}, t)\right] r_{x} d \bar{\Gamma}\left(r_{x}, z_{x}\right),
\end{gathered}
$$

where

$$
\left\{\begin{array}{l}
u_{n}^{{ }^{*} \mathrm{c}}(\bar{\xi}, \bar{x}, \tau-t)=\frac{1}{4 \pi} \int_{0}^{2 \pi} u^{*}(\bar{\xi}, \bar{x}, \theta, \tau-t) \cos n \theta d \theta \\
u_{n}^{*_{s}}(\bar{\xi}, \bar{x}, \tau-t)=\frac{1}{4 \pi} \int_{0}^{2 \pi} u^{*}(\bar{\xi}, \bar{x}, \theta, \tau-t) \sin n \theta d \theta
\end{array}\right.
$$

Similarly, we obtain:

$$
\begin{gathered}
\frac{1}{\pi} \int_{0}^{2 \pi} \sin n \theta_{\xi} d \theta_{\xi} \int_{\Gamma} u^{*}(\xi, x, \tau, t) q(x, t) d \Gamma_{x}= \\
=\int_{\bar{\Gamma}}\left[u_{n}^{* c}(\bar{\xi}, \bar{x}, \tau-t) q_{n}^{s}(\bar{x}, t)-u_{n}^{*_{s}}(\bar{\xi}, \bar{x}, \tau-t) q_{n}^{c}(\bar{x}, t)\right] r_{x} d \bar{\Gamma}\left(r_{x}, z_{x}\right), \quad n=\overline{1, \infty} .
\end{gathered}
$$

In view of (8), (13) and (14), (5) can be rewritten as: 


$$
\left\{\begin{array}{l}
\mathrm{c}(\bar{\xi}) \mathrm{u}_{\mathrm{n}}^{\mathrm{c}}(\bar{\xi}, \tau)+\int_{\mathrm{t}_{0}}^{\tau} \mathrm{dt} \int_{\bar{\Gamma}}\left\{\mathrm{u}_{\mathrm{n}}^{*_{\mathrm{c}}}(\bar{\xi}, \overline{\mathrm{x}}, \tau-\mathrm{t}) \mathrm{q}_{\mathrm{n}}^{\mathrm{c}}(\overline{\mathrm{x}}, \mathrm{t})+\mathrm{u}_{\mathrm{n}}^{*_{\mathrm{s}}}(\bar{\xi}, \overline{\mathrm{x}}, \tau-\mathrm{t}) \mathrm{q}_{\mathrm{n}}^{\mathrm{s}}(\overline{\mathrm{x}}, \mathrm{t})-\right. \\
\left.-\mathrm{u}_{\mathrm{n}}^{\mathrm{c}}(\overline{\mathrm{x}}, \mathrm{t}) \mathrm{q}_{\mathrm{n}}^{*_{\mathrm{c}}}(\bar{\xi}, \overline{\mathrm{x}}, \tau-\mathrm{t})-\mathrm{u}_{\mathrm{n}}^{\mathrm{s}}(\overline{\mathrm{x}}, \mathrm{t}) \mathrm{q}_{\mathrm{n}}^{*_{\mathrm{s}}}(\bar{\xi}, \overline{\mathrm{x}}, \tau-\mathrm{t})\right\} \mathrm{r}_{\mathrm{x}} \mathrm{d} \bar{\Gamma}\left(\mathrm{r}_{\mathrm{x}}, \mathrm{z}_{\mathrm{x}}\right)= \\
=\int_{\Omega} \rho \mathrm{c} \mathrm{u}_{0}(\mathrm{x}) \frac{1}{4 \pi} \int_{0}^{2 \pi} \mathrm{u}^{*}\left(\bar{\xi}, \theta_{\xi}, \mathrm{x}, \tau-\mathrm{t}_{0}\right) \cos \mathrm{n} \theta_{\xi} \mathrm{d} \theta_{\xi} \mathrm{d} \Omega_{\mathrm{x}}- \\
-\int_{\mathrm{t}_{0}}^{\tau} \mathrm{dt} \int_{\Omega} \mathrm{f}(\mathrm{x}, \mathrm{t}) \frac{1}{4 \pi} \int_{0}^{2 \pi} \mathrm{u}^{*}\left(\bar{\xi}, \theta_{\xi}, \mathrm{x}, \tau-\mathrm{t}_{0}\right) \cos \mathrm{n} \theta_{\xi} \mathrm{d} \Omega_{\mathrm{x}}, \\
\mathrm{c}(\bar{\xi}) \mathrm{u}_{\mathrm{n}}^{\mathrm{s}}(\bar{\xi}, \tau)+\int_{\mathrm{t}_{0}}^{\tau} \mathrm{dt} \int_{\bar{\Gamma}}\left\{\mathrm{u}_{\mathrm{n}}^{*_{\mathrm{c}}}(\bar{\xi}, \overline{\mathrm{x}}, \tau-\mathrm{t}) \mathrm{q}_{\mathrm{n}}^{\mathrm{s}}(\overline{\mathrm{x}}, \mathrm{t})-\mathrm{u}_{\mathrm{n}}^{*_{\mathrm{s}}}(\bar{\xi}, \overline{\mathrm{x}}, \tau-\mathrm{t}) \mathrm{q}_{\mathrm{n}}^{\mathrm{c}}(\overline{\mathrm{x}}, \mathrm{t})-\right. \\
\left.-\mathrm{u}_{\mathrm{n}}^{\mathrm{s}}(\overline{\mathrm{x}}, \mathrm{t}) \mathrm{q}_{\mathrm{n}}^{*_{\mathrm{c}}}(\bar{\xi}, \overline{\mathrm{x}}, \tau-\mathrm{t})+\mathrm{u}_{\mathrm{n}}^{\mathrm{c}}(\overline{\mathrm{x}}, \mathrm{t}) \mathrm{q}_{\mathrm{n}}^{*_{\mathrm{s}}}(\bar{\xi}, \overline{\mathrm{x}}, \tau-\mathrm{t})\right\} \mathrm{r}_{\mathrm{x}} \mathrm{d} \bar{\Gamma}\left(\mathrm{r}_{\mathrm{x}}, \mathrm{z}_{\mathrm{x}}\right)= \\
=\int_{\Omega} \rho \mathrm{c} \mathrm{u}_{0}(\mathrm{x}) \frac{1}{4 \pi} \int_{0}^{2 \pi} \mathrm{u}^{*}\left(\bar{\xi}, \theta_{\xi}, \mathrm{x}, \tau-\mathrm{t}_{0}\right) \sin \mathrm{n} \theta_{\xi} \mathrm{d} \theta_{\xi} \mathrm{d} \Omega_{\mathrm{x}}- \\
-\int_{\mathrm{t}_{0}}^{\tau} \mathrm{dt} \int_{\Omega} \mathrm{f}(\mathrm{x}, \mathrm{t}) \frac{1}{4 \pi} \int_{0}^{2 \pi} \mathrm{u}^{*}\left(\bar{\xi}, \theta_{\xi}, \mathrm{x}, \tau-\mathrm{t}_{0}\right) \sin \mathrm{n} \theta_{\xi} \mathrm{d} \Omega_{\mathrm{x}},
\end{array}\right.
$$

where $\bar{\xi} \in \bar{\Gamma}, \mathrm{n}=\overline{0, \infty}$.

So, for an axially symmetric domain the boundary integral equation (5), that is three-dimensional in four-dimensional Minkowski space, is reduced to a system of two-dimensional singular integral equations (15). Integral equations (15) are equations of the second kind for the Neumann problem, equations of the first kind for the Dirichlet problem, mixed type for the mixed problem.

Let's note that if the boundary conditions, initial conditions and source function are also axially symmetric (in cylindrical coordinate system they are independent of $\theta_{x}$ ), the system (15) is reduced to one two-dimensional in four-dimensional Minkowski space singular integral equation. Accordingly, if the conditions of the problem can be represented as a segment of the Fourier series with $\mathrm{n}$ members, the problem is reduced to a system of $2 \mathrm{n}$ two-dimensional singular integral equations.

\section{3. The fundamental solution for axially symmetric domain} we have:

Let's investigate the fundamental solution $\mathrm{u}_{\mathrm{n}}^{*_{\mathrm{c}}}, \mathrm{u}_{\mathrm{n}}^{*_{\mathrm{s}}}$ from (15). Taking into account (10), (11),

$$
\begin{aligned}
& u_{\mathrm{n}}^{*_{\mathrm{c}}}(\bar{\xi}, \bar{x}, \tau-\mathrm{t})=\frac{1}{\pi} \int_{0}^{2 \pi} \mathrm{u}^{*}(\xi-\mathrm{x}, \tau-\mathrm{t}) \cos n \theta \mathrm{d} \theta=-\frac{(\rho \mathrm{c})^{1 / 2}}{\pi(4 \pi \mathrm{k}(\tau-\mathrm{t}))^{3 / 2}} \mathrm{H}(\tau-\mathrm{t}) \times \\
& \quad \times \int_{0}^{2 \pi} \exp \left\{-\frac{\rho \mathrm{c}}{4 \mathrm{k}(\tau-\mathrm{t})}\left(\mathrm{r}_{\xi}{ }^{2}+\mathrm{r}_{\mathrm{x}}{ }^{2}-2 \mathrm{r}_{\xi} \mathrm{r}_{\mathrm{x}} \cos \theta+\left(\mathrm{z}_{\xi}-\mathrm{z}_{\mathrm{x}}\right)^{2}\right)\right\} \cos n \theta \mathrm{d} \theta .
\end{aligned}
$$

Due to the fact that:

$$
\begin{gathered}
\int_{0}^{2 \pi} e^{a \cos \theta} \sin n \theta d \theta=0, \\
\int_{0}^{2 \pi} e^{a \cos \theta} \cos n \theta d \theta=2 \pi I_{n}(a),
\end{gathered}
$$

where $I_{n}(z)$ is a modified $n$-th Bessel function, expressions $u_{n}^{*_{s}}, u_{n}^{*_{c}}$ from (15) are the coefficients in the Fourier decomposition for fundamental solution, that can be written as: 


$$
\left\{\begin{array}{l}
\mathrm{u}_{\mathrm{n}}^{*_{\mathrm{c}}}(\bar{\xi}, \bar{x}, \tau-\mathrm{t})=-\frac{(\rho \mathrm{c})^{1 / 2} \cdot 2}{(4 \pi \mathrm{k}(\tau-\mathrm{t}))^{3 / 2}} \mathrm{H}(\tau-\mathrm{t}) \times \exp \left\{-\frac{\rho \mathrm{c}}{4 \mathrm{k}(\tau-\mathrm{t})}\left(\mathrm{r}_{\xi}{ }^{2}+\mathrm{r}_{\mathrm{x}}{ }^{2}+\left(\mathrm{z}_{\xi}-\mathrm{z}_{\mathrm{x}}\right)^{2}\right)\right\} \times \\
\times \mathrm{I}_{\mathrm{n}}\left(\frac{\rho \mathrm{c} \mathrm{r}_{\mathrm{x}} \mathrm{r}_{\xi}}{2 \mathrm{k}(\tau-\mathrm{t})}\right), \\
\mathrm{u}_{\mathrm{n}}^{\mathrm{*}_{\mathrm{s}}}(\bar{\xi}, \bar{x}, \tau-\mathrm{t})=0 .
\end{array}\right.
$$

Define $\mathrm{q}_{\mathrm{n}}^{*_{\mathrm{c}}}$ and $\mathrm{q}_{\mathrm{n}}^{*_{\mathrm{s}}}$. Because $\mathrm{q}^{*}(\xi-\mathrm{x}, \tau-\mathrm{t})=\mathrm{k} \frac{\partial \mathrm{u}^{*}}{\partial \mathrm{n}_{\mathrm{x}}}$, then $\mathrm{q}^{*}=\mathrm{k}\left(\frac{\partial \mathrm{u}^{*}}{\partial \mathrm{r}_{\mathrm{x}}} \mathrm{n}_{\mathrm{r}_{\mathrm{x}}}+\frac{\partial \mathrm{u}^{*}}{\partial \mathrm{z}_{\mathrm{x}}} \mathrm{n}_{\mathrm{z}_{\mathrm{x}}}\right)$. For axially symmetric domain $\hat{n}_{\mathrm{x}}=\hat{\mathrm{r}}_{\mathrm{x}} \mathrm{n}_{\mathrm{r}_{\mathrm{x}}}+\hat{z}_{\mathrm{z}_{\mathrm{x}}}$ and we obtain:

$$
\left\{\begin{array}{l}
\frac{\partial u^{*}}{\partial r_{x}}=\frac{\partial u^{*}}{\partial|\xi-x|} \cdot \frac{\partial|\xi-x|}{\partial r_{x}}=-\frac{(\rho c)^{1 / 2}}{(4 \pi k(\tau-t))^{3 / 2}} \frac{(-2)|\xi-x| \rho c}{4 k(\tau-t)} H(\tau-t)\left(2 r_{x}-2 r_{\xi} \cos \theta\right) \times \\
\times \exp \left(-\frac{|\xi-x|^{2} \rho c}{4 k(\tau-t)}\right), \\
\frac{\partial u^{*}}{\partial z_{x}}=\frac{\partial u^{*}}{\partial|\xi-x|} \cdot \frac{\partial|\xi-x|}{\partial z_{x}}=-\frac{(\rho c)^{1 / 2}}{(4 \pi k(\tau-t))^{3 / 2}} \frac{(-2)|\xi-x| \rho c}{4 k(\tau-t)} H(\tau-t) 2\left(z_{\xi}-z_{x}\right)(-1) \times \\
\times \exp \left(-\frac{|\xi-x|^{2} \rho c}{4 k(\tau-t)}\right) .
\end{array}\right.
$$

This system, using (17), can be rewritten as:

$$
\left\{\begin{array}{l}
\frac{\partial u^{*}}{\partial r_{x}}=\left(A_{n}+B_{n} \cos \theta+C_{n} \cos ^{2} \theta\right) e^{a \operatorname{css} \theta}, \\
\frac{\partial u^{*}}{\partial z_{x}}=\left(A_{q}+B_{q} \cos \theta\right) e^{a \operatorname{cs} \theta} \theta
\end{array}\right.
$$

where

$$
\begin{aligned}
& A_{n}=- \frac{(\rho c)^{1 / 2}}{(4 \pi k(\tau-t))^{3 / 2}} \frac{(-2) \rho c\left(r_{\xi}^{2}+r_{x}^{2}+\left(z_{\xi}-z_{x}\right)^{2}\right)}{4 k(\tau-t)} H(\tau-t) \times \\
& \times \exp \left(-\frac{\rho c}{4 k(\tau-t)}\left(r_{\xi}^{2}+r_{x}^{2}+\left(z_{\xi}-z_{x}\right)^{2}\right)\right), \\
& B_{n}=-\frac{(\rho c)^{1 / 2}}{(4 \pi k(\tau-t))^{3 / 2}} \frac{(-2) \rho c}{4 k(\tau-t)} H(\tau-t)\left(-2 r_{\xi}\left(r_{\xi}^{2}+r_{x}^{2}+\left(z_{\xi}-z_{x}\right)^{2}\right)-2 r_{\xi} r_{x}^{2}\right) \times \\
& \quad \times \exp \left(-\frac{\rho c}{4 k(\tau-t)}\left(r_{\xi}^{2}+r_{x}^{2}+\left(z_{\xi}-z_{x}\right)^{2}\right)\right), \\
& C_{n}=-\frac{(\rho c)^{1 / 2}}{(4 \pi k(\tau-t))^{3 / 2}} \frac{(-2) \rho c}{4 k(\tau-t)} H(\tau-t) 4 r_{\xi}^{2} r_{x} \times \\
& \quad \times \exp \left(-\frac{\rho c}{4 k(\tau-t)}\left(r_{\xi}^{2}+r_{x}^{2}+\left(z_{\xi}-z_{x}\right)^{2}\right)\right),
\end{aligned}
$$




$$
\begin{gathered}
A_{q}=-\frac{(\rho c)^{1 / 2}}{(4 \pi k(\tau-t))^{3 / 2}} \frac{(-2) \rho c\left(r_{\xi}^{2}+r_{x}^{2}+\left(z_{\xi}-z_{x}\right)^{2}\right)}{4 k(\tau-t)} H(\tau-t)(-2)\left(z_{\xi}-z_{x}\right) \times \\
\quad \times \exp \left(-\frac{\rho c}{4 k(\tau-t)}\left(r_{\xi}^{2}+r_{x}^{2}+\left(z_{\xi}-z_{x}\right)^{2}\right)\right), \\
B_{q}=-\frac{(\rho c)^{1 / 2}}{(4 \pi k(\tau-t))^{3 / 2}} \frac{(-2) \rho c\left(-2 r_{\xi} r_{x}\right)}{4 k(\tau-t)} H(\tau-t)(-2)\left(z_{\xi}-z_{x}\right) \times \\
\quad \times \exp \left(-\frac{\rho c}{4 k(\tau-t)}\left(r_{\xi}^{2}+r_{x}^{2}+\left(z_{\xi}-z_{x}\right)^{2}\right)\right) .
\end{gathered}
$$

Applying obvious trigonometric transformations and entering notation:

$$
\mathrm{a}=\frac{\rho \mathrm{c} \mathrm{r}_{\mathrm{x}} \mathrm{r}_{\xi}}{2 \mathrm{k}(\tau-\mathrm{t})}
$$

we find $\mathrm{q}_{\mathrm{n}}^{{ }^{*} \mathrm{c}}(\bar{\xi}, \overline{\mathrm{x}}, \tau-\mathrm{t})$ and $\mathrm{q}_{\mathrm{n}}^{{ }_{\mathrm{s}}}(\bar{\xi}, \overline{\mathrm{x}}, \tau-\mathrm{t})$ :

$$
\left\{\begin{array}{l}
\mathrm{q}_{\mathrm{n}}^{* \mathrm{c}}(\bar{\xi}, \bar{x}, \tau-\mathrm{t})=2 \pi \mathrm{n}_{\mathrm{r}} \mathrm{k}_{\mathrm{x}}\left\{\mathrm{A}_{\mathrm{n}} \mathrm{I}_{\mathrm{n}}\left(\frac{\rho \mathrm{c} \mathrm{r}_{\mathrm{x}} \mathrm{r}_{\xi}}{2 \mathrm{k}(\tau-\mathrm{t})}\right)+\mathrm{B}_{\mathrm{n}} \frac{1}{2}\left(\mathrm{I}_{\mathrm{n}+1}(\mathrm{a})+\mathrm{I}_{\mathrm{n}-1}(\mathrm{a})\right)+\right. \\
\left.+\frac{1}{2} \mathrm{C}_{\mathrm{n}}\left(\mathrm{I}_{\mathrm{n}}(\mathrm{a})+\frac{1}{2} \mathrm{I}_{\mathrm{n}+2}(\mathrm{a})+\frac{1}{2} \mathrm{I}_{\mathrm{n}-2}(\mathrm{a})\right)\right\}+2 \pi \mathrm{n}_{\mathrm{z}_{\mathrm{x}}} \mathrm{k}\left\{\mathrm{A}_{\mathrm{q}} \mathrm{I}_{\mathrm{n}}(\mathrm{a})+\mathrm{B}_{\mathrm{q}} \frac{1}{2}\left(\mathrm{I}_{\mathrm{n}+1}(\mathrm{a})+\mathrm{I}_{\mathrm{n}-1}(\mathrm{a})\right)\right\} \\
\mathrm{q}_{\mathrm{n}}^{* \mathrm{~s}}(\bar{\xi}, \bar{x}, \tau-\mathrm{t})=0 .
\end{array}\right.
$$

Thus, we find the explicit formulas for finding functional coefficients (components) of the Fourier decomposition of fundamental solutions $\mathrm{u}^{*}, \mathrm{q}^{*}$ from (15). Consequently, (15) defines a system of integral equations, solving this system we find the boundary values of functions (or $u_{n}^{s}$, or $q_{n}^{s}$ ). Obtaining the Fourier series of the form (6), we find the unknown boundary values. Then, using the integral representation (5), we can get the complete solution to the problem in the axially symmetric domain.

\section{Conclusions}

The work is devoted to investigation of non-stationary initial boundary value problem of thermal conductivity with not axially symmetric right part of the equation and boundary conditions for axially symmetric domain in Minkowski space.

Using the methods of boundary integral equations and the Fourier decomposition, the problem is reformulated as an infinite set of two-dimensional singular integral equations for coefficients of expansion. Fundamental solutions, used in the integral representation of the solution in the domain and on the border, are built in the explicit form and studied.

Reducing of the initial four-dimensional boundary problem to the set of two -dimensional equations by taking into account the axisymmetric of the domain is important for the problem of constructing of the effective numerical boundary element method for Real-Time settlements of behavior of structures.

Interpretation of initial-boundary problem as a boundary value problem in Minkowski space allows to use the results of the article for solving problems in axially symmetric domains variable over time.

The ideas and methods of this study can be applied in very similar problems in linear thermoelasticity. Such vector problems are related and imply a transition to a matrix description. According to the results of the article, such transition is possible. In addition, using the Kirchhoff transformation, the proposed approach can be generalized for problems with spatial inhomogeneous coefficient of thermal conductivity and for nonlinear problems (with power nonlinearity of thermal conductivity coefficient). 


\section{References}

[1] Kartashov, E., Kudinov, V. (2012). Analytical theory of heat conduction and thermoelasticity. Librocom, 656.

[2] Lurie, A. I. (2010). Theory of Elasticity. Springer Science \& Business Media, 1050. doi: $10.1007 / 978-3-540-26455-2$

[3] Maceri, A. (2010). Theory of Elasticity, 1st Edition. Springer Science \& Business Media, 716. doi: 10.1007/978-3-642-11392-5

[4] Zarubin, V., Kyvurkin, G. (2002). Mathematical models of thermal mechanics. Moscow: Fizmatlit, 168.

[5] Wrobel, L. C., Aliabadi, M. H. (2002). The Boundary Element Method, New York: John Wiley \& Sons, 1066.

[6] Constanda, C., Doty, D., Hamill, W. (2016). Boundary Integral Equation Methods and Numerical Solutions. New York: Springer, 232. doi: 10.1007/978-3-319-26309-0

[7] Schanz, M., Steinbach, O. (2007). Boundary Element Analysis - Mathematical Aspects and Applications. Lecture Notes in Applied and Computational Mechanics, 352. doi: 10.1007/978-3-540-47533-0

[8] Zrazhevsky, G., Zrazhevska, V. (2016). Obtaining and investigation of the integral representation of solution and boundary integral equation for the non-stationary problem of thermal conductivity. Eureka: Physics and Engineering, 6, 53-58. doi: 10.21303/2461-4262.2016.00216

[9] Reddy, J. N. (2006). An Introduction to the Finite Element Method, 3rd Edition. McGraw Hill, 672.

[10] Chaskalovic, J. (2008). Finite Elements Methods for Engineering Sciences. Springer Verlag, 267. 